\title{
Light optical and Ultrastructural Characteristics of the Maral Liver with Chronic Dicrocoeliasis
}

\author{
Darzhigitova Albina Koshanovna ${ }^{1}$, Shapekova Nelya Lukpanovna ${ }^{2}$, Karlygash Aubakirova ${ }^{2}$, Ainur Koigeldinova ${ }^{3}$, Tynykulov
} Marat Korganbekovich ${ }^{2}$, Kaisagaliyeva Gulzhakhan ${ }^{4}$

${ }^{1}$ Department of General Biology and Genomics, L.N. Gumilyov Eurasian National University, Nur-Sultan, Kazakhstan; ${ }^{2}$ Department of Biotechnology and Microbiology, L.N. Gumilyov Eurasian National University, Nur-Sultan, Kazakhstan; ${ }^{3}$ Department of Veterinary Medicine, Shakarim State University, Semey, Kazakhstan; ${ }^{4}$ Department of Biology and Ecology, M. Utemisov West Kazakhstan University, Uralsk, Kazakhstan

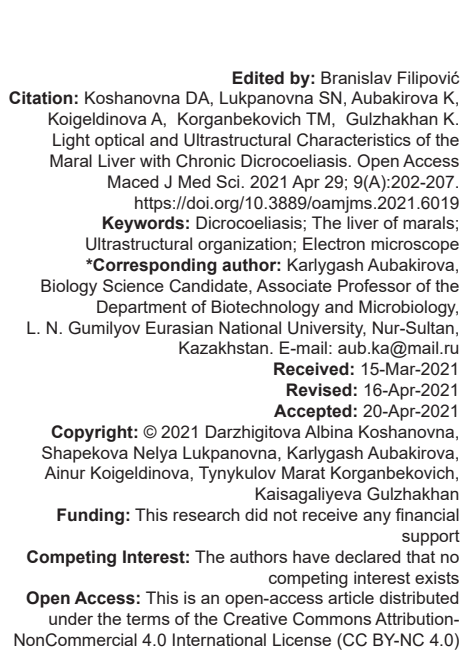

\section{Abstract}

BACKGROUND: Dicrocoeliasis is caused by trematode Dicrocoelium lanceatum from the family Dicrocoeliidae, a parasite in the bile ducts of the liver of domestic and wild animals. Dicrocoeliasis mainly affects sheep, cattle, camels, zebu, deer, fallow deer, argali, less often - horses, donkeys, dogs, rabbits, hares and bears, as well as humans Dicrocoeliasis of ruminants is widespread across the whole Kazakhstan. Invasive diseases represent a significant obstacle in the development of domestic maral breeding, among which trematodoses, and particularly dicrocoeliasis of maral play a major role.

AIM: The aim of the research was to study the influence of dicrocoelia on the ultrastructural organization of the live of maral.

MATERIALS AND METHODS: For examination under electron microscope, biopsy pieces of liver tissue of mara were fixed in $2.5 \%$ solution of glutaraldehyde with post-fixation in $1 \%$ solution of osmium tetroxide, conducted according to a conventional method, and enclosed in epon. Semi-thin and ultra-thin sections were prepared on the ultra-microtome Leica. Semi-thin sections were stained with methylene blue, azure 2, and studied at a highresolution light optical level. The ultra-thin sections were contrasted with uranyl acetate and lead citrate according Reynolds method and examined under electron microscope Libra 120 (C. Zeiss)

RESULTS: The light optical examination of half-thin sections revealed that the morphological pattern of pathological changes in liver tissue was polymorphic, even within a single hepatic lobe.

CONCLUSIONS: In the liver of maral infected with chronic dicrocoeliasis, dystrophic and destructive pathological changes developed in all the cellular structures of the hepatic lobules: In the form of plethora and vast enlargement of sinusoids, vacuolar and lipodegeneration of hepatocytes, destruction of the hepatic tissue with edema, hemorrhages in the appearance of cells associated with inflammation, and the deposition of hematin crystals.

\section{Introduction}

Maral farming is a new, fast developing and high-income field of animal husbandry. It represents a high value for the economy of Kazakhstan. The production of velvet antlers has a strategic importance for the agro-industrial sector, socioeconomic status of the significant part of population in rural areas depends on export of the antlers products.

East Kazakhstan region is the main place of production and export of the products of velvet antlers deer farming in Kazakhstan. At present, there are 24 farms of different forms of ownership, which specialize in the maral farming. The climate and natural conditions of the region are favorable for breeding and farming of maral. The objective at this moment is to improve the current system of velvet antlers deer farming and to increase the production. To solve this problem, it is necessary for this field to reach an active development, that is, to improve the quality and the quantity of products while keeping a good control of the overall antler deer population. Part of this solution is to improve the conditions of keeping the animals and conditions of feeding but also it is important to provide an epizootic well-being of maral farms by protecting them from infectious and invasive diseases.

According to domestic researchers, outbreaks of invasive dicrocoeliasis are still present in different climatic zones of the country. Dicrocoeliasis is prevalent in the steppe, semi-desert and forest-steppe zone of the country, where it becomes a great burden to the livestock economy. Incidents of the infected livestock in southern regions of Kazakhstan reach $80 \%$. Beginning of the pasture season and mid-summer is the season with the highest rates of infection. The disease begins to clinically manifest itself at an intensity of invasion (II) of more than 300 specimens in animals older than 1.5 years. As the animals age, the intensity of invasion increases, reaching more than 25 thousand specimens, with the extent of 
invasion in sheep $80-100 \%$ and in cattle 40-98\%. In the meantime, economic damage is caused by a decrease in the volume and quality of dairy and meat productivity, and also by the costs of medical and preventive measures. This is due to lack of efficiency and untimely therapeutic and preventive measures, which leads to an increase in the formation of trematode biotopes of intermediate and additional hosts [1], [2], [3].

Usenbaev et al., found a significant infestation with dicrocoeliidae of sheep - up to $78.6 \%$ and cattle - $45.0 \%$. In this case, dicrocoelia formed associations with other parasites inside the animals' body, consisting of two, three, four, or five subparts. Most of the times, there was a four-membered association with the following component composition: Fasciola + dicrocelia + strongylata + Eimeria (33.3\%) [4]. Kaspakbaev et al. reported that the invasion of farm animals with helminthiases in Kazakhstan is quite high, while official veterinary statistics does not correspond to the reality due to lack of control over anthelmintic activity, due to sharp decline in the production of livestock products and a reduction of the animal population [5]. Erbolat and Suleimenov, based on the results of research in seven oblasts of Kazakhstan, noted various mixed invasions in animals, including those that are formed from various ratios of Fasciola and dicrocoelia. The authors also report that the diversity of helminths in farm animals and the predominance of individual helminth groups in associations with certain climatic and geographical zones of Kazakhstan make it extremely difficult to control them. The solution of the problem of helminthiases is complicated by the presence of the variety of climatic zones and subzones on the territory of Kazakhstan, by the diversity of groups and types of helminthic pathogens, by the lack of an arsenal of antihelminthic products and products of biological protection for animals, by the disaggregation of farms, and by the predominance of private property in the agricultural sector [6].

An important role in the evaluation of the epizootic situation of dicrocoeliasis and in the helminthological assessment of pastures belongs to data on the survival of eggs and larvae of dicrocoelia in the external environment. Invasive elements of dicrocoelia have a great survival rate in the external environment. Hence, when the moistened areas dry, the eggs of the dicrocoelia retain viability for 10-12 months. They remain vial for a long time even at the temperature of $43-45^{\circ} \mathrm{C}$. Dicrocoelium larvae retain the invasiveness in the body of intermediate and additional hosts for up to 3 years or more (Tverdokhlebov and Ayupov, 1980) [7]. In each climatic zone, the extent and intensity of the dicrocoeliasis invasion for each individual animal species varies significantly. The situation with dicrocoeliasis is poor in many areas of East Kazakhstan, where the infection of ruminants with dicrocoelia sometimes reaches $100 \%$ with an invasive intensity of 30 thousand copies. Foothill regions, mountains, and steppe regions are especially at risk of dicrocoeliasis.
It was found that maral dicrocoeliasis invasions during the past 10 years were recorded more often - in 68.1 (2006) and 82.0\% (2016) of animals [7], [8], [9].

Thus, the intensive spread of the disease is facilitated by the presence of a wide range of definitive, intermediate, and additional hosts of dicrocoelia, and also by the stability of invasive elements in the external environment.

The main pathogenic effect of dicrocoelia is concentrated on the liver: Clogged and damaged bile ducts, poor digestion, and bile secretion. In addition, a big part of the clinical picture has a toxic and allergic effect. The pathogenesis is distinguished by the absence of a period of hematogenous migration. Cercariae of the parasite escape the intestine through the outlets of bile ducts and undergo maturation first in the small, and then in the large bile ducts. Pathomorphology bears resemblance of parasitic cholangitis, expressed in the appearance of thick whitish crimped cords of the affected ducts under the capsule and on the cut of the liver. When invasion is intensive, the disease progresses similarly to biliary cirrhosis: The liver is enlarged, the surface is shagreen or tuberous form, brown, dense, and is penetrated by a network of connective tissue strands of the affected bile ducts, from which a mucous yellow-brown liquid containing parasites is secreted. The gallbladder is enlarged and contains viscous bile with parasites [10].

Publications on dicrocoeliasis in Kazakhstan are limited to the studies about the distribution of parasites among large and small cattle, their effect on animal productivity, hematologic indices, pathoanatomical changes in organs, and various economic damage. Methods of treatment and prophylactic measures of the disease are also described. However, there are no available research data from a detailed study on the effects of dicrocoelia on the maral liver at the histological and ultrastructural level. Due to lack of data concerning the effect of dicrocelia on the maral organism and the demand to develop a research-based system of antidicrocoeliasis measures, our research was dedicated toward this field.

\section{Materials and Methods}

For examination under electron microscope, biopsy pieces of liver tissue of maral were fixed in a $2.5 \%$ solution of glutaraldehyde with post-fixation in $1 \%$ solution of osmium tetroxide, conducted according to a conventional method, and enclosed in epon. Semithin and ultra-thin sections were prepared on the ultramicrotome Leica. Semi-thin sections were stained with methylene blue, azure 2, and studied at a highresolution light optical level. The ultra-thin sections were contrasted with uranyl acetate and lead citrate 
according Reynolds method and examined under electron microscope Libra 120 (C. Zeiss).

\section{Research Results and Discussion}

The light optical examination of half-thin sections revealed that the morphological pattern of pathological changes in liver tissue was polymorphic, even within a single hepatic lobe. The liver tissue located closer to the center of the lobule was characterized by plethora, capillarization of sinusoids, a sharp expansion and edema of perinosinoid and sinusoidal space, the appearance of lymphocytes and macrophages, and vacuolar dystrophy of hepatocytes (Figure 1).

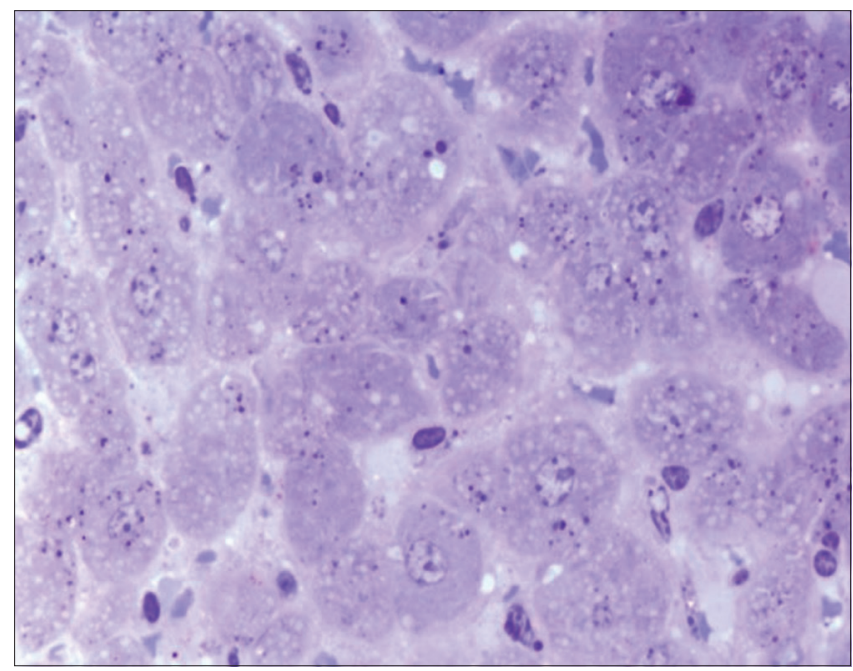

Figure 1: Expansion and plethora of sinusoids. Vacuolar degeneration of hepatocytes. Semi-thin section. Staining with methylene blue, azure $2 \times 400$

When observed under electron microscope, the hepatocyte nuclei were characterized by a high euchromatin content, a primary location of the condensed chromatin, or contained coarse accumulations of condensed chromatin (Figure 2).

Perinuclear space is narrow. Numerous large mitochondria had a round or oval shape. Some of them tool giant forms. The cristae are few, some portion of the crista is finely fragmented, a matrix of low electron density, finely grained. The tubules of the granular endoplasmic reticulum were located without strict orientation, less often in an orderly parallel series and were characterized by the degranulation of fixed ribosomes. The agranular endoplasmic reticulum was represented by small vesicles and long thin tubules. The granules of glycogen were absent. Lipid inclusions were present with signs of recycling. Numerous autophagosomes were present in the cytoplasm (Figure 3).

Elements of the Golgi complex occupied a small area and were vacuolized. The biliary surface contained rare short microvilli. A complete reduction of microvilli was also noted (Figure 4).

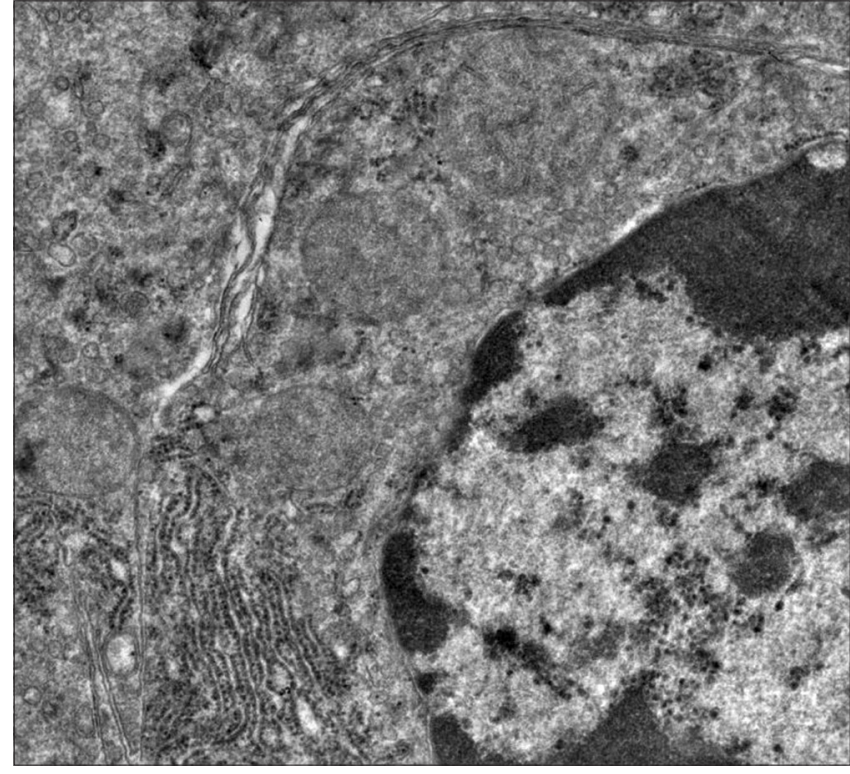

Figure 2: The nucleus of a hepatocyte with condensed chromatin. Mitochondria with fragmented cristae. Canals of a granular endoplasmic reticulum with degranulation of fixed ribosomes. Visible long tubules of an agranular reticulum. Electronogram

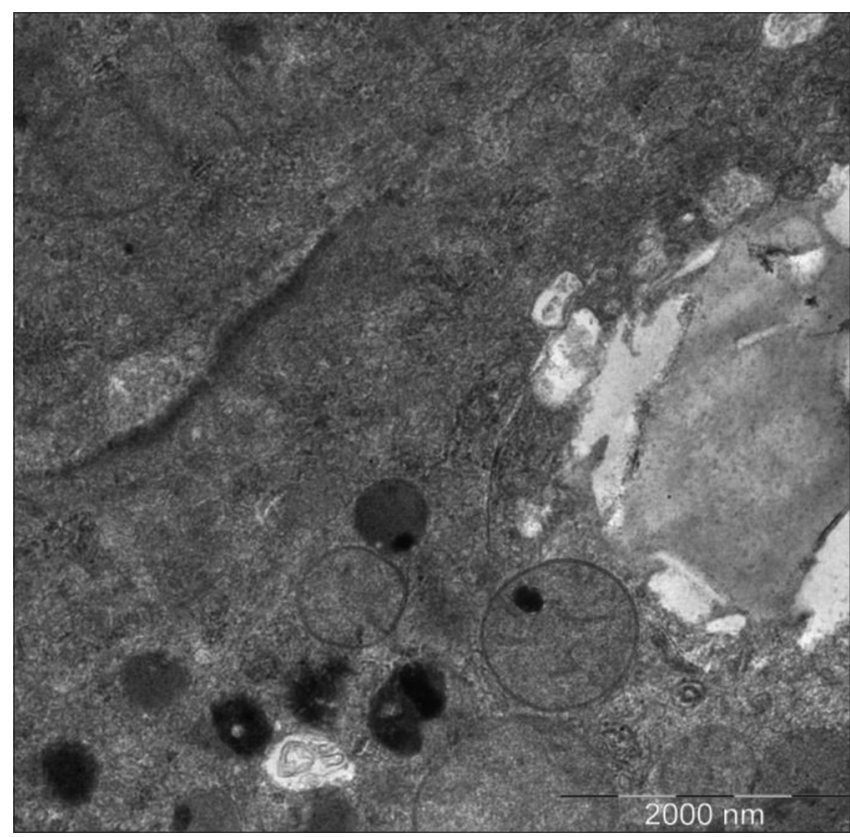

Figure 3: Lipid inclusions with signs of recycling. Lysosomes. Electronogram

The destruction of the end plates was observed (Figure 5). At the same time, the intercellular spaces were significantly enlarged and connected the lumen of the bile capillaries with sinusoids (Figure 6).

The sinusoidal surface of hepatocytes was smooth, indistinct, and partially destroyed (Figure 7).

The space of Disse was expanded and almost merged with the space of sinusoids, caused by a destruction of the endothelium. Occasionally, closer to the periphery of the lobules, there were regions of hepatocytes with pronounced lipodystrophy, complete destruction of the liver structure, hemorrhages, and swelling of the interstitial tissue (Figure 8). 


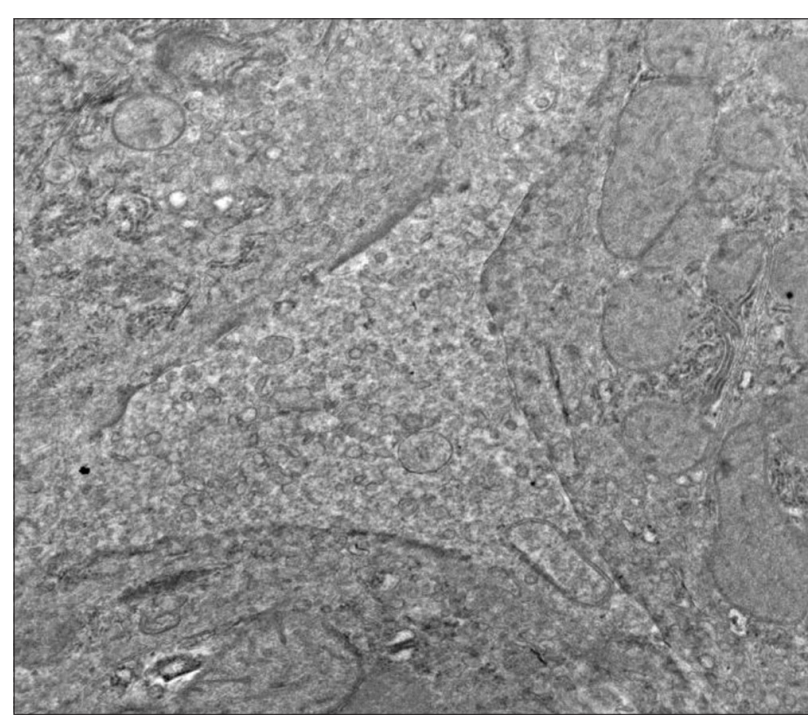

Figure 4: Expansion of the lumen of the bile capillary with reduction of microvilli. Vacuolization of the Golgi complex. Electronogram

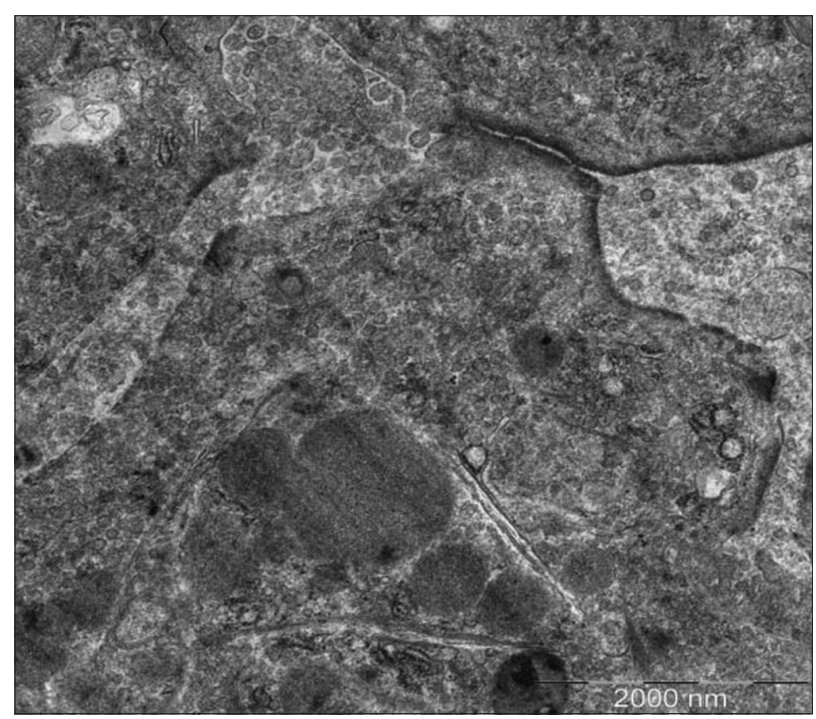

Figure 5: Disruption of the end plates. Electronogram

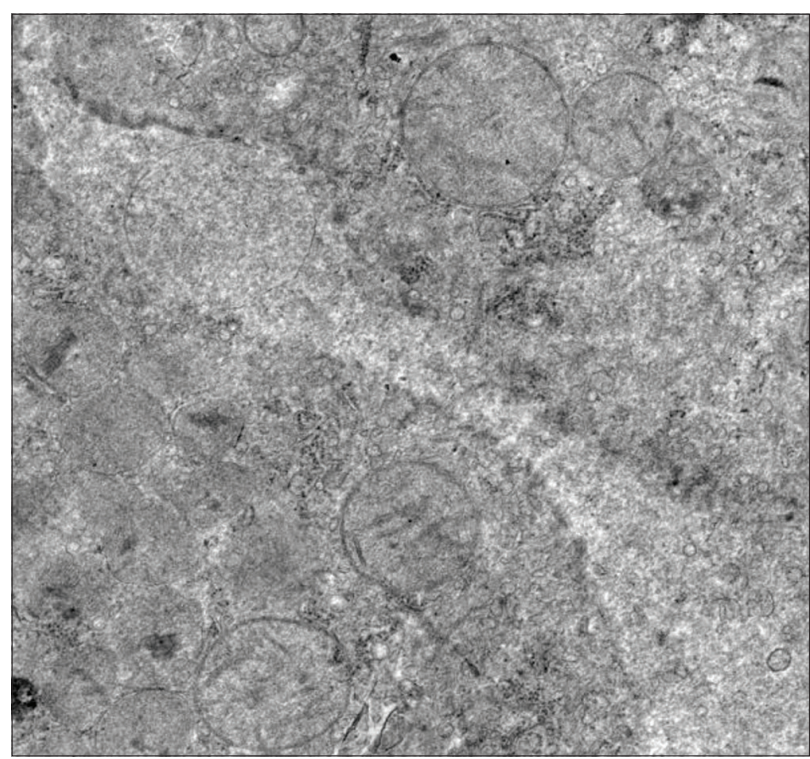

Figure 6: Significant enlargement of intercellular cracks. Electronogram
Lipid inclusions with signs of recycling had round shape and were distinctly of large sizes, up to a complete replacement of the hepatocytes' cytoplasm. Closer to triads the liver structure was destroyed; the separately located hepatocytes were located in the edematous and fibrous connective tissue. In addition, there could be seen clusters of lymphocytes, freely floating red blood cells, regions of hemorrhage, and numerous long crystals in the form of rhombic plates and needles, belonging to the structures of the disbalanced process of hemoglobin pigmentation. Blood vessels of triads were significantly enlarged. Epithelium of cholangiol was high and light with signs of vacuolar

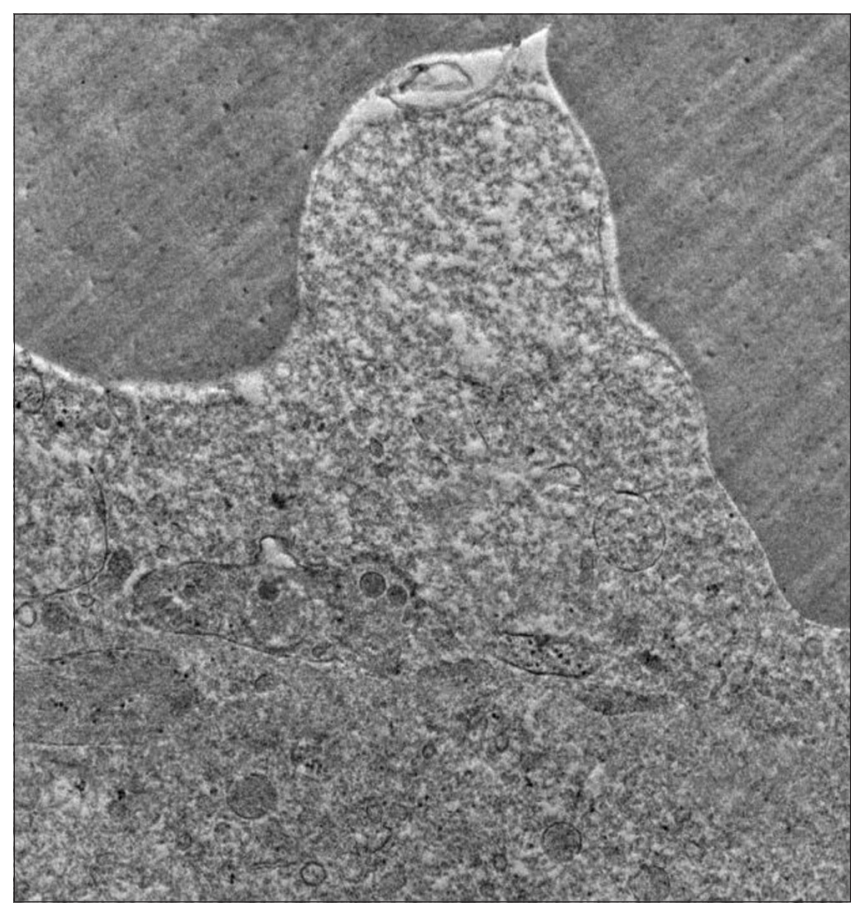

Figure 7: Plasmalemma destruction of the sinusoidal surface of hepatocyte. Destruction of the ultrastructure of endothelium. Flocculent masses in the space of Disse and sinusoidal space. Electronogram

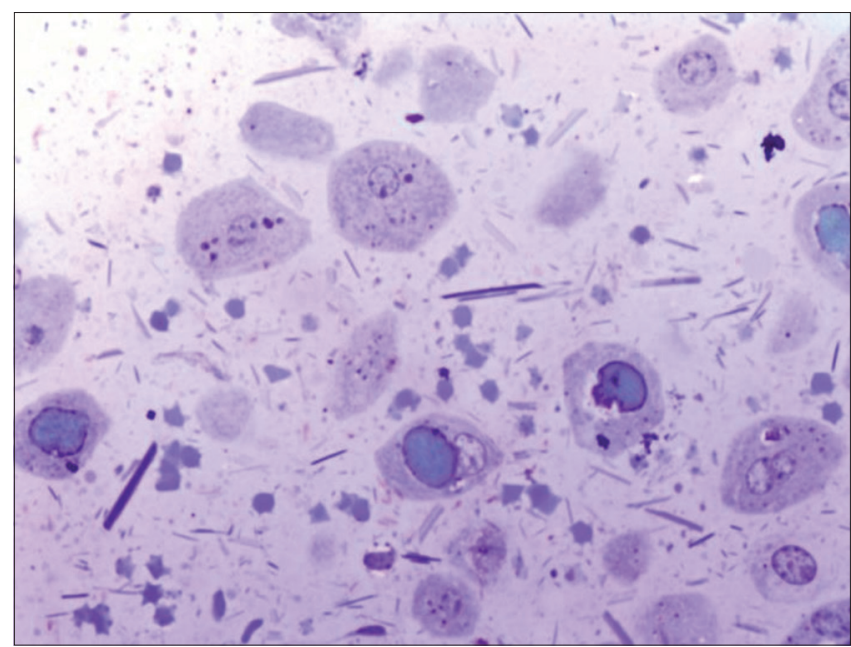

Figure 8: Hepatocytes with large inclusions of fat. Liver destruction, edema, hemorrhage. Semi-thin section. Staining with methylene blue, azure $2 \times 400$ 
dystrophy. Intermittent bile ducts proliferated in the form of narrow elongated structures, the epithelium nuclei looked dark pycnotic. Epithelium of cholangiol was elevated and light, with signs of vacuolar dystrophy. Intermittent bile ducts proliferated in the form of narrow elongated structures, the epithelium nuclei looked dark and pyknotic. Occasionally completely necrotic hepatic lobules were also observed.

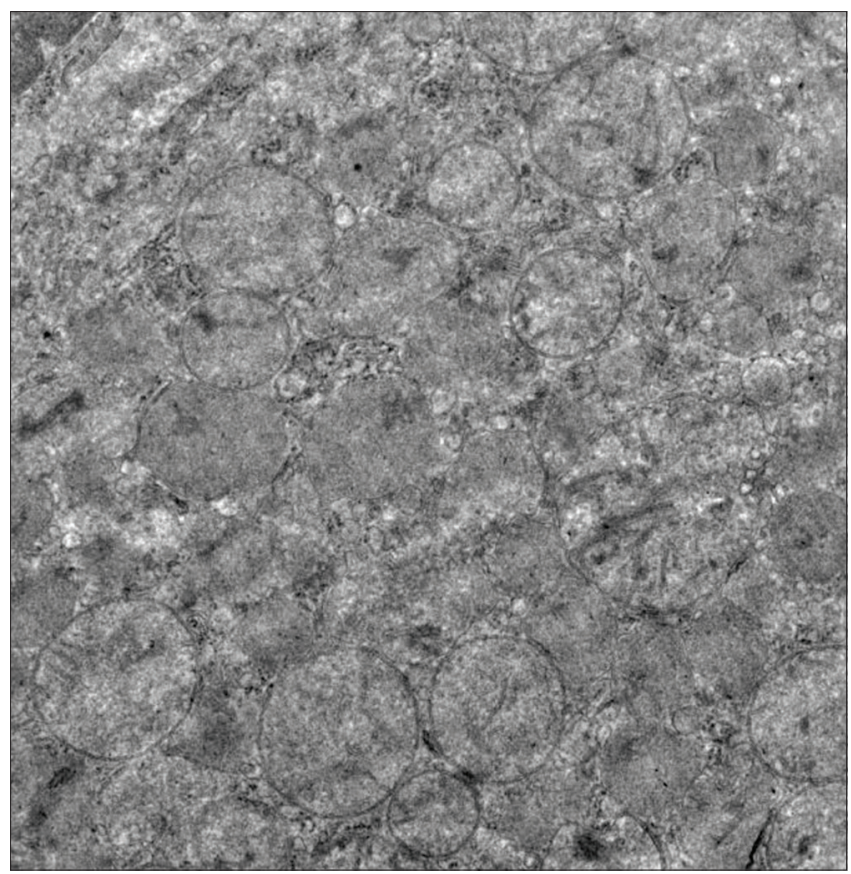

Figure 9: Destruction of mitochondria. Electronogram

During the examination under electron microscope, the signs of chromatin condensation in the nuclei of hepatocytes were becoming more apparent, up to the karyopyknosis. Part of the mitochondria was in a state of destruction (Figure 9).

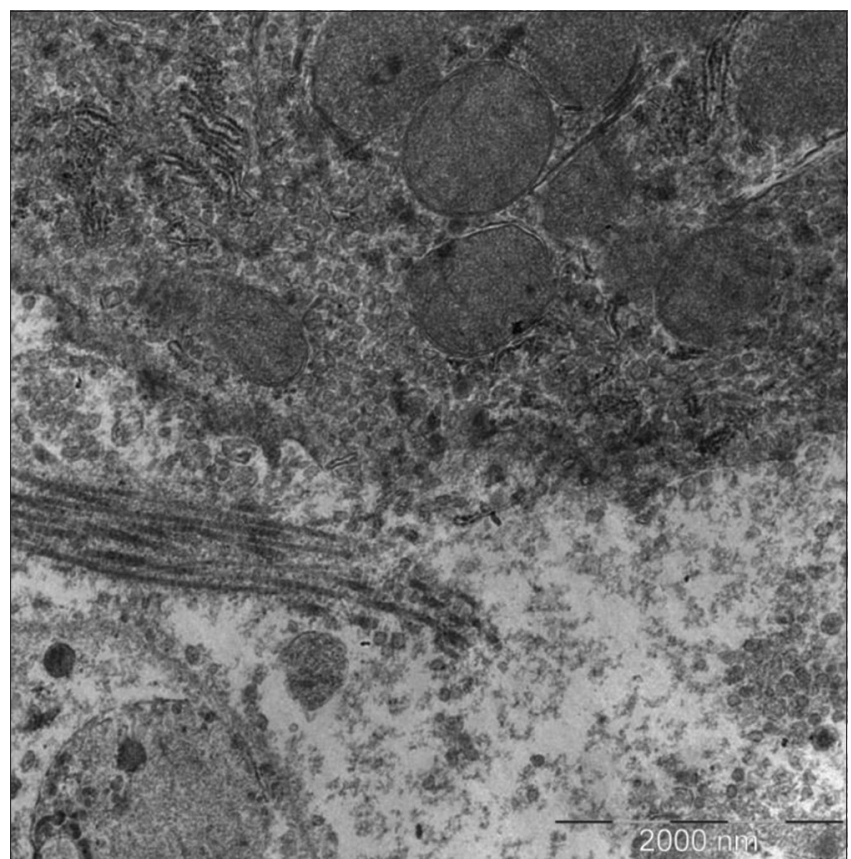

Figure 10: Bundles of collagen fibrils and apoptotic bodies in the space of Disse. Electronogram
The membranes of the granular endoplasmic reticulum were homogenized. The regions of the enlarged bile capillaries were connected with the space of sinusoids. Sinusoidal surface of hepatocytes was destroyed. The space of Disse and sinusoidal space were filled with fragments of destroyed hepatocytes, light-colored apoptotic "balls," Kaunsilmen bodies, erythrocytes, unstructured flaky mass, and bundles of collagen fibrils (Figures 10 and 11).

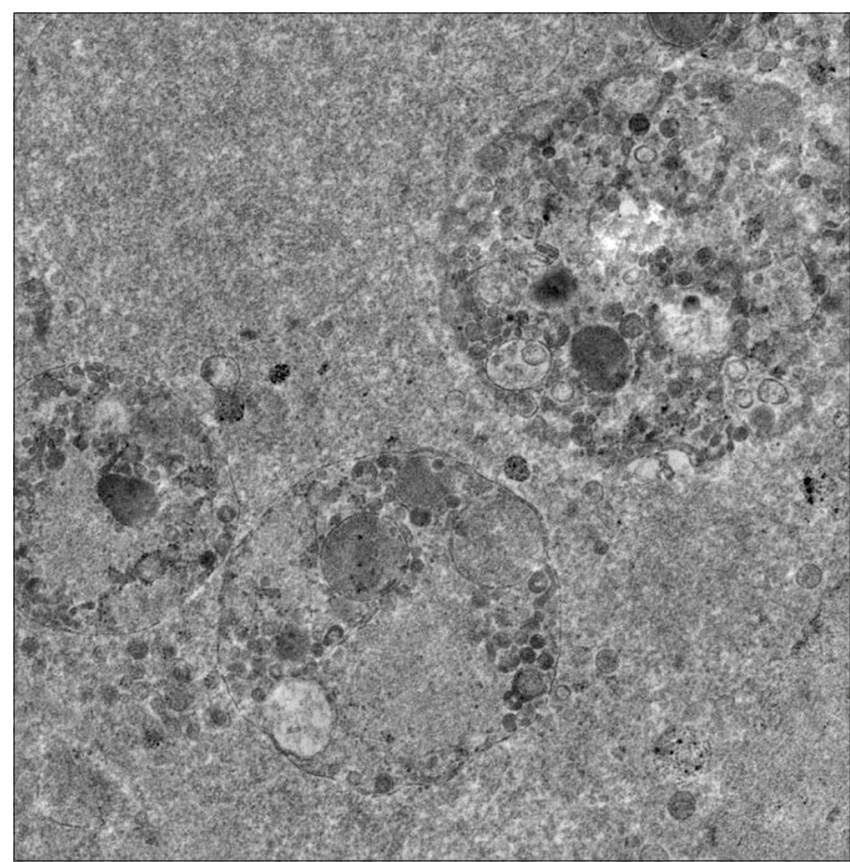

Figure 11: Apoptosis of hepatocytes. Kaunsilmen bodies. Electronogram

Morphologically altered hepatocytes by necrosis were surrounded by structureless masses. In the region of necrosis of hepatocytes and hemorrhages, there were present accumulations of rhomboid crystals of hematin (Figure 12).

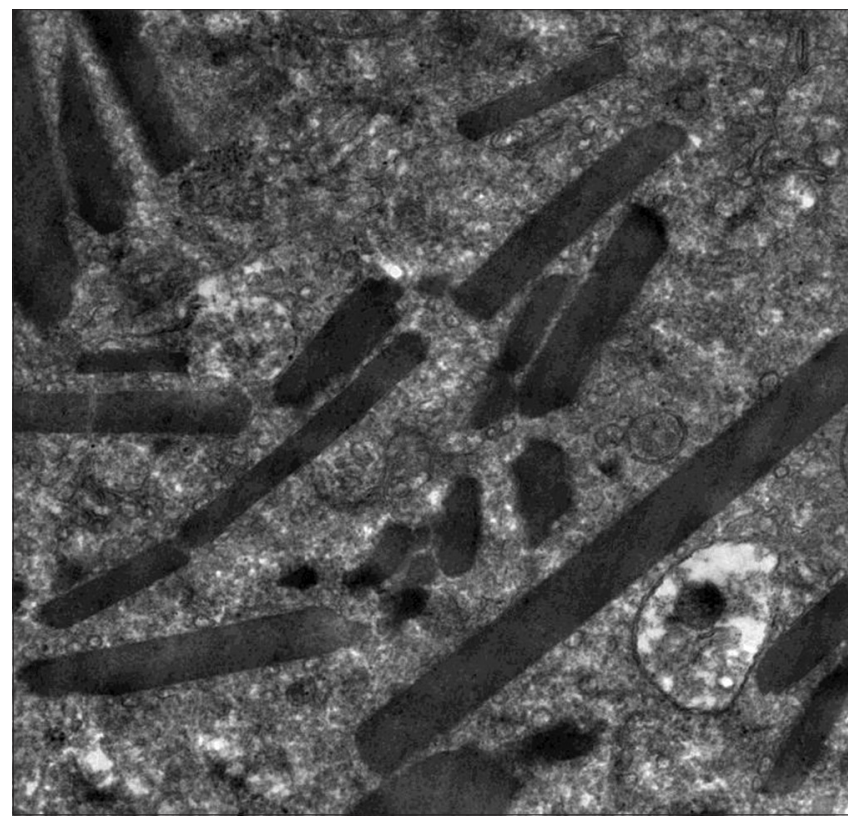

Figure 12: Crystals of hematin among the destroyed cellular material. Electronogram 


\section{Conclusions}

In the liver of maral infected with chronic dicrocoeliasis, dystrophic and destructive pathological changes developed in all the cellular structures of the hepatic lobules: In the form of plethora and vast enlargement of sinusoids, vacuolar and lipodegeneration of hepatocytes, destruction of the hepatic tissue with edema, hemorrhages, in the appearance of cells associated with inflammation, and the deposition of hematin crystals.

\section{References}

1. Shuklina E.V. Features of epizootology and the system of therapeutic and prophylactic measures for associative invasion of marals. Dis Can Vet Sci. 2007:22.

2. Lunitsyn VG, Terentyev VI. Epizootic Situation on Invasive Diseases of Marals. Organomorphology and Prevention of Animal Diseases. Barnaul: Conference to the $55^{\text {th }}$ Anniversary of the Altai State Agrarian University (Barnaul, December 25, 1998); 2000. p. 65.

3. Lunitsyn VG. The Main Parasitoses of Marals, Schemes of their Prevention and Therapy. Barnaul: Azbuka; 2011. p. 236.
4. Usenbaev AE, Suleimenov MZ, Zhumakhanov B. Association of Parasites of the Digestive Tract of Sheep and Cattle in the South of Kazakhstan. Almaty: Scientific Support of Measures to Combat Infectious and Invasive Diseases of Farm Animals in Kazakhstan Sat Scientific Works Dedicated to the $75^{\text {th }}$ Anniversary of the Institute, Almaty; 2000. p. 409-20. https://doi. org/10.25276/2410-1257-2018-4-66-68

5. Kaspakbaev AS, Kuznetsov VI, Ananiev OP. Helminthiasis of the Gastrointestinal Tract and Experiments on their Prevention in Sheep. Modern Measures to Combat Infectious and Invasive Diseases of Farm Animals in Kazakhstan. Almaty: Collection of Scientific Papers, Almaty; 2003. p. 44-7.

6. Erbolat KM, Suleimenov MZ. Prevention of Mixed Helminthiasis in Ruminants. Modern Measures to Combat Infectious and Invasive Diseases of Farm Animals in Kazakhstan. Almaty: Collection of Scientific Papers; 2003.

7. Tverdokhlebov PT, Ayupov KV. Dicroceliosis of Animals Moscow, VO: "Agropromizdat"; 1988. p. 174.

8. Burlinski P, Janiszewski P, Kroll A, Gonkowski S. Parasitofauna in the gastrointestinal tract of the cervids (Cervidae) in Northern Poland. Acta Vet Belgrade. 2011:61:269-82. https://doi. org/10.2298/avb1103269b

9. Bermagambetova A, Shabdarbaeva GS. Infection of Ruminants with Dicroceliosis in the South of Kazakhstan. Materials of the International Scientific-practical Conference. In: Asanov NG, editor. Modern Problems of Combating Especially Dangerous, Exotic and Zooanthroponous Diseases of Animals, dedicated to the $70^{\text {th }}$ Anniversary, Almaty; 2012. p. 226-30.

10. Shmakova O. Distribution of Dicroceliosis in Populations of Cattle and Marals in the Altai Territory. Bulletin of the Ulyanovsk State Agricultural Academy; 2019. p. 70-4. https://doi. org/10.18286/1816-4501-2019-1-70-74 